\title{
Carbohydrate-associated epitope-based anti-cancer drugs and vaccines*
}

\author{
Gregory Lee ${ }^{1,2}$, Cheng-Yuan Huang ${ }^{2}$, Song-Nan Chow ${ }^{3}$, Chin-Hsiang Chien ${ }^{4}$ \\ ${ }^{1}$ UBC Center for Reproductive Health, University of British Columbia, Vancouver, Canada \\ ${ }^{2}$ Vancouver BioTech Ltd., Vancouver, Canada \\ ${ }^{3}$ Department of Obstetrics/Gynecology, National Taiwan University Hospital, Taipei, Chinese Taipei \\ ${ }^{4}$ Institute of Biochemistry and Molecular Biology, National Yang Ming University, Taipei, Chinese Taipei \\ Email: cyglee@yahoo.com
}

Received 27 June 2013; revised 28 July 2013; accepted 15 August 2013

Copyright (C) 2013 Gregory Lee et al. This is an open access article distributed under the Creative Commons Attribution License, which permits unrestricted use, distribution, and reproduction in any medium, provided the original work is properly cited.

\begin{abstract}
RP215 is one of the three thousand monoclonal antibodies (Mabs) which were generated against the OC3-VGH ovarian cancer cell line. RP215 was shown to react with a carbohydrate-associated epitope located specifically on glycoproteins, known as CA215, from cancer cells. Further molecular analysis by matrix adsorption laser desorption/ionization time-of-flight mass spectrometry (MALDI-TOF MS) revealed that CA215 consists mainly of immunoglobulin super-family (IgSF) proteins, including immunoglobulins, Tcell receptors, and cell adhesion molecules, as well as several other unrelated proteins. Peptide mappings and glycoanalysis were performed with CA215 and revealed high-mannose and complex bisecting structures with terminal sialic acid in $\mathrm{N}$-glycans. As many as ten $\mathrm{O}$-glycans, which are structurally similar to those of mucins, were also identified. In addition, two additional $\mathrm{O}$-linked glycans were exclusively detected in cancerous immunoglobulins but not in normal $B$ cell-derived immunoglobulins. Immunizations of mice with purified CA215 resulted in the predominant generation of RP215-related Mabs, indicating the immunodominance of this carbohydrate-associated epitope. Anti-idiotype (anti-id) Mabs of RP215, which were generated in the rat, were shown to contain the internal images of the carbohydrate-associated epitope. Following immunizations of these anti-id Mabs in mice, the resulting anti-anti-id (Ab3) responses in mice were found to be immunologically similar to that of RP215. Judging from these observations, anti-id Mabs, which carry the internal image of the RP215specific epitope, may be suitable candidates for anticancer vaccine development in humans.
\end{abstract}

${ }^{*}$ Conflict of interest: Gregory Lee is a co-founder of Vancouver BioTech Ltd.
Keywords: Anti-Cancer Drugs; Anti-Cancer Vaccines; Anti-Idiotype; CA215; Carbohydrate-Associated Epitope; Immunodominance; RP215

\section{INTRODUCTION}

Among the monoclonal antibodies (Mabs) which were generated against the OC-3-VGH ovarian cancer cell line, RP215 was shown to react with a carbohydrate-associated epitope detected in cancerous glycoproteins, known as CA215. CA215 consists mainly of immunoglobulin superfamily (IgSF) proteins, including antigen receptors such as immunoglobulins, T-cell receptors, and several other related molecules, as well as unrelated mucins [1]. During the last decade, efforts have been made to characterize RP215 Mab and its cognate antigen, CA215. Cancerous immunoglobulins are the predominant molecules among CA215 that are recognized by RP215 through this specific carbohydrate-associated epitope [2].

Several experimental observations led us to conclude that the RP215-specific epitope in CA215 is carbohydrate-associated. For example, a significant loss of RP215 epitope activity was observed when CA215 was treated with mild sodium periodate solution $(10-100$ $\mathrm{mM}$ ) at neutral $\mathrm{pH}$, extreme heat (e.g. $100^{\circ} \mathrm{C}$ for $5 \mathrm{~min}$ ), culturing of cancer cells in serum-free medium, or incubation at extreme $\mathrm{pH}(\leq 2.0$ or $\geq 12.0)[2-4]$. This is in contrast to normal human immunoglobulin $\mathrm{G}$ (IgG), of which the immunoactivity remained stable under the above conditions [2-4].

The ability of cancer cells to express immunoglobulins has been known for decades [5]. These cancerous immunoglobulins have been found to be essential for the growth/proliferation of cancer cells $[4,6]$. With RP215 as the unique immunoprobe, numerous biological and immunological studies were performed to explore the mechanisms of the action of cancerous immunoglobulins. 
A significant progress has been made regarding our current understanding of cancer cell-expressed immunoglobulins and their potential roles [7]. By means of terminal deoxynucleotidyl transferase dUTP nick end labeling (TUNEL) apoptosis assays, it was clearly demonstrated that RP215 or antibodies against immunoglobulins or T-cell receptors, induced apoptosis in all studied cultured cancer cells, irrespective of their tissue origins [8]. By immunohistochemical studies, immunoglobulins with the RP215 epitope can be readily detected on the surface of cancer cells [9]. Consequently, complement-dependent cytotoxicity (CDC) reactions can also be induced by antibodies against a variety of different classes or subclasses of immunoglobulins, including IgG, immunoglobulin $\mathrm{M}(\operatorname{IgM})$, immunoglobulin A (IgA), $\lambda$ light chain or $\kappa$ light chain $[4,8]$. CDC reactions can also be induced similarly with RP215 [8,9]. This led us to suggest the possibility that both RP215 and anti-antigen receptor antibodies are involved in affecting similar gene regulation patterns within cancer cells. This was indeed the case when gene regulation studies were performed with more than a dozen selected genes which are essential for the growth and proliferation of cancer cells. Excellent correlations were obtained between the gene regulation patterns of these selected genes and treatment with RP215 or anti-antigen receptors [7]. Furthermore, both RP215 and anti-antigen receptors exhibited similarly strong influences on the gene expressions of tolllike receptors $[7,10]$. In particular, additional gene regulation studies revealed that genes of selected toll-like receptors, TLR-2, TLR-3, TLR-4, and TLR-9, were significantly affected by RP2 15 and antibodies against antigen receptors [7]. Since toll-like receptors are key components of cellular innate immunity, this observation strongly suggests the involvement of cancerous immunoglobulins or antigen receptors in the innate immune system of cancer cells [7,10].

Peptide mapping and glycoanalysis of purified CA215 were performed in an attempt to elucidate the molecular structure of the carbohydrate-associated epitope recognized by RP215 [1] and are highlighted in this review. In addition, the immunological nature of RP215-specific epitope was also investigated through studies of more antiCA215 Mabs and anti-idiotype (anti-id) Mabs against RP215. The possibility of using carbohydrate epitopebased anti-cancer vaccines was evaluated based on these experimental observations [11].

\section{BIOCHEMICAL AND IMMUNOLOGICAL NATURE OF THE CARBOHYDRATE-ASSOCIATED EPITOPE}

\subsection{Structural Analysis of Carbohydrate Epitope Recognized by RP215}

Efforts were made to identify and elucidate the structures of the RP215-specific carbohydrate-associated epitope. These included structural analysis of the N-linked and O-linked oligosaccharides in CA215 [1]. Initially, the shed media from two cultured cancer cell-lines, OC-3VGH (ovarian) and C-33A (cervical), were collected separately and purified for CA215 by RP215-affinity chromatography. To obtain affinity-purified cancerous IgG (designated as CA215-D), affinity-purified CA215 was further subjected to purification by anti-human IgG affinity chromatography (designated as CA215-D). The affinity-purified CA215 samples were desalted and cleaned of borate, permethylated, and analyzed either by nano spray ionization-linear ion trap mass spectrometry (NSILTQ/MSn) or by matrix-assisted laser desorption/ ionization time-of-flight mass spectrometry (MALDI-TOF MS). N-linked glycan profiling revealed unusually high mannose structures, as well as terminal N-glycolylnueraminic acids, both of which do not appear in normal human IgG [12]. On the other hand, several cancer-associated mucin-type O-glycans were found in CA215, including core 1 to 3 based structures such as sialyl-T, sialyl-Tn, and di-sialyl-T antigens, and Lewis antigens such as $\mathrm{Le}^{\mathrm{a}}, \mathrm{Le}^{\mathrm{b}}, \mathrm{Le}^{\mathrm{x}}, \mathrm{SLe}^{\mathrm{x}}$, and $\mathrm{Le}^{\mathrm{y}}$. The potential pathways for the synthesis of the carbohydrate-associated epitope recognized by RP215 have been elucidated by the studies of glycosyl-transferases which are involved in O-glycan synthesis (Gao. Y. and Brockhausen, I., personal communication). Results of the comparative profiles of permethylated O-linked glycans from five different CA215 samples are presented in Table 1. From the structural analysis of O-linked oligosaccharides identified for CA215 , it can be demonstrated that cancerous IgG (CA215D) can be attached with two different distinct O-linked oligosaccharides: GalNAc $\mathrm{Gal}_{1} \mathrm{NeuAc}_{1}$ and GalNAc $\mathrm{Gal}_{1}$ $\mathrm{NeuAc}_{2}$. These two O-linked oligosaccharides are unique to cancerous $\operatorname{IgG}$, but absent in normal human IgG [12]. It remains to be elucidated to see if these two O-linked oligosaccharides are structurally linked to the RP215specific carbohydrate-associated epitope.

Following further analysis with glycosylation site peptide mapping, the potential N-glycosylation and O-glycosylation sites along the peptides sequences were identified by Protein BLAST service. Among the ten glycopeptides which were detected from CA215, eight were shown to be homologous to the Fab and/or Fc fragments of human immunoglobulin heavy chains. All the analytical results of the ten glycopeptides are summarized in Table 2. These experimental analyses strongly suggested that immunoglobulin heavy chains are the predominant molecular species in CA215 [1,2]. In addition, the RP215specific epitope activity of CA215 was found to be $\mathrm{pH}$-dependent. At $\mathrm{pH} 12$, deglycosylation of CA215 may result in loss of RP215 epitope activity. 
Table 1. Comparative profiles of permethylated O-linked glycans of human IgG and five different CA215 samples.

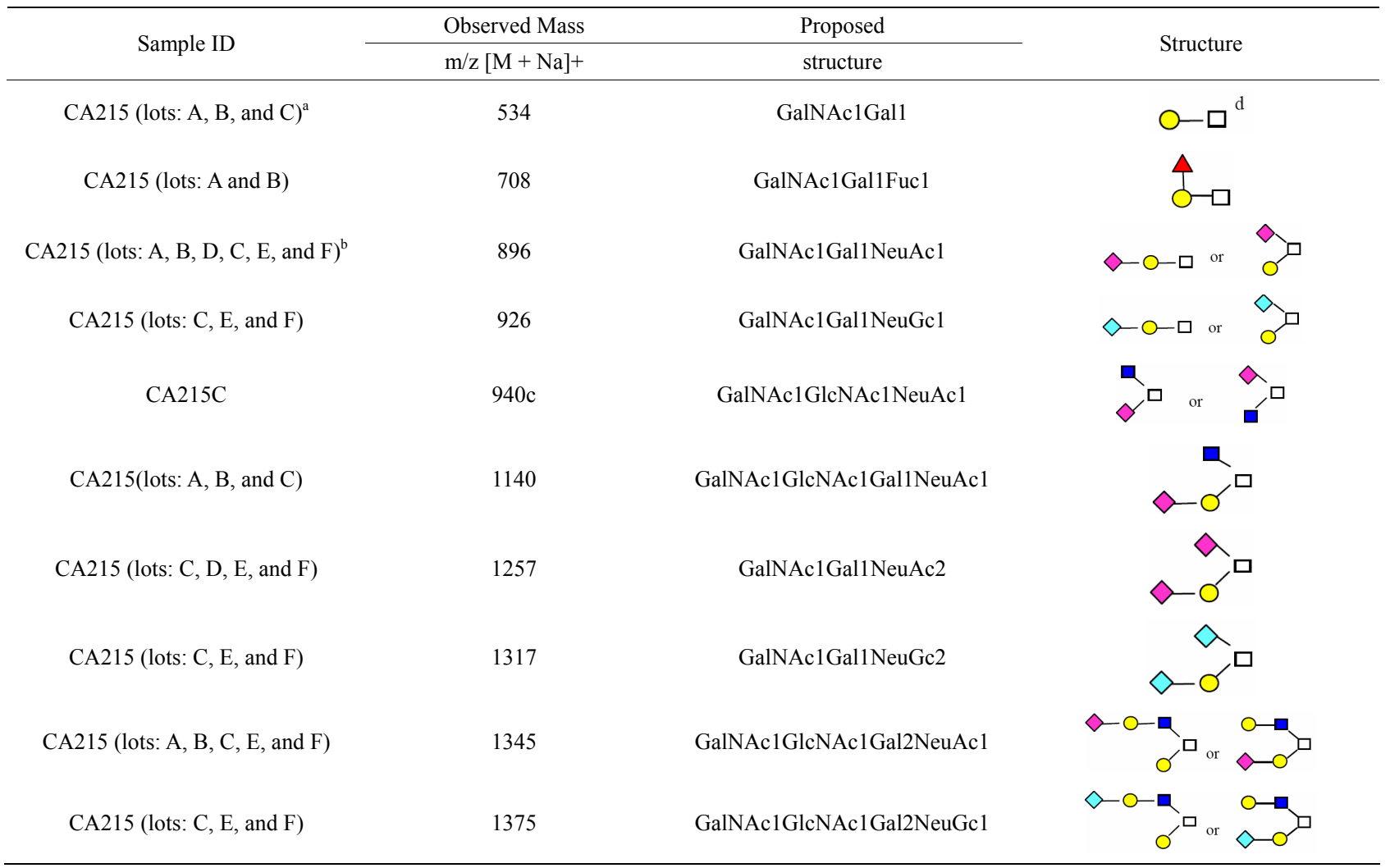

${ }^{\mathrm{a} C A} 215$ lots A, B and C were from OC-3-VGH ovarian cancer cells (CA215-OC-3) lots A and B were obtained through acid elution, whereas lots C, D, E and F were obtained through elution with $3 \mathrm{M}$ urea. ${ }^{\mathrm{b}}$ Lot CA215D was obtained by an additional purification of urea-eluted CA215 (S15K-100425) with goat anti-human. IgG affinity column followed by the same analysis (CA215D is designated as affinity-purified cancerous IgG). CA215 lots E and F were from C-33A cervical cancer cells (CA215-C33A). ${ }^{\mathrm{c}}$ Detected by MALDI-TOF MS method but not found by NSI-MS method. ${ }^{\mathrm{d}} \mathrm{N}-\mathrm{ace}$-tylgalactosamine ( $\square$ ), $\mathrm{N}$-acetylglucosamine $(\bullet)$, Fucose $(\boldsymbol{\Delta})$, Galactose $(\bullet)$, N-acetylneuraminic acid $(\bullet)$ and N-glycolylneuraminic acid $(\bullet)$. [Obtained from reference \#1 with permission].

Table 2. N-linked and O-linked glycosylation site mappings of CA215. Fc refers to the constant region of the immunoglobulins. Fab represents the variable region of the immunoglobulins. Lot CA215C (urea-eluted) was used for this analysis. The source protein BLAST service was: http://blast.ncbi.nlm.nih.gov/Blast.cgi.

\begin{tabular}{|c|c|c|}
\hline Accession number & Peptide detected $^{\mathrm{a}}$ & Peptide sequence homology of proteins (\%) \\
\hline I. CAC12842.1 & 1. EEQFNSTFR & Immunoglobulin heavy chain $(\mathrm{Fc})(100 \%)$ \\
\hline II. CAA04843.1 & 2. EEQFNSTYR & Immunoglobulin heavy chain $(\mathrm{Fc})(100 \%)$ \\
\hline III. AAB60643.2 & 3. LSVPTSEWQR & Cathepsin S (100\%) \\
\hline \multirow[t]{5}{*}{ IV. AAK68690.1 } & 4. FTCLATNDAGDSSK & Hemicentin $(100 \%)$ \\
\hline & & Titin (100\%) \\
\hline & & Palladin isoform $4(92 \%)$ \\
\hline & & LRN4 (78\%) \\
\hline & & (IgSF proteins) \\
\hline V. AAD38158.1 & 5. DTLMISR & Immunoglobulin heavy chain $(\mathrm{Fc})(100 \%)$ \\
\hline VI. AAC39746.2 & 6. GYLPEPVTVTWNSGTLTNGVR & Immunoglobulin heavy chain (Fab) $(90 \%)$ \\
\hline VII. AAN76042.1 & 7. SVSLTCMINGFYPSDISVEWEK & Immunoglobulin heavy chain $(\mathrm{Fc})(90 \%)$ \\
\hline VIII. CAJ75462.1 & 8. QSSGLYSLSSVVSVTSSSQPVTCNV & Immunoglobulin heavy chain (Fab and $\mathrm{Fc})(100 \%)$ \\
\hline \multirow{3}{*}{ IX. ABY48864.2 } & 9. VYTMGPPREELSSR & Immunoglobulin heavy chain $(\mathrm{Fc})(98 \%)$ \\
\hline & & IgA variable region $(89 \%)$ \\
\hline & & $\operatorname{IgM}(98 \%)$ \\
\hline \multirow[t]{3}{*}{ X. NP_001139647.1 } & 10. TFPSVR & Zinc finger protein 414 isoform I (100\%) \\
\hline & & Forkhead box protein C2 (100\%) \\
\hline & & Immunoglobulin heavy chain variable region $(83 \%)$ \\
\hline
\end{tabular}

a; Bold letters indicate glycosylation sites [Obtained from reference \#1 with permission]. 


\subsection{Molecular and Immuno-Characteristics of Cancerous Immunoglobulins}

The molecular and immunological nature of cancerous immunoglobulins was further investigated initially through reverse transcription polymerase chain reaction (RT-PCR) to determine the primary structures of cancerous $\operatorname{IgG}$ and IgA at the DNA and protein levels. In the constant regions of the heavy chains, the IgG and IgA detected in cancer cells were identical to those of normal human B cells with homology greater than $98 \%[2,13]$. Studies were conducted to investigate if the glycosylations in cancerous immunoglobulins have any alterations in their biochemical and immunological properties as compared to those of normal human IgG. As stated previously, cancerous IgG was isolated by two-step affinity chromatography from the OC-3-VGH ovarian cancer cell line. Unexpectedly, the isolated cancerous IgG (CA215D in Table 1) exhibits extremely low immunoactivity when compared to that of normal human IgG. Apparently, the aberrant glycosylations have altered the tertiary structure of the cancerous IgG and subsequently its immunological properties [3].

\subsection{Immunodominance of RP215-Specific Epitope in CA215}

Attempts were made to generate additional Mabs against affinity-purified CA215. Some of the epitopes recognized by the new Mabs were conformational, while others were linear, similar to that recognized by RP215. When compared with Mabs against CA215, the amino acid sequence homology to RP215's Fab regions ranged from $100 \%$ to $65 \%$ for the five generated Mabs which were designated as RCA-10, RCA-100, RCA-104, RCA-110 and RCA-111, respectively [14]. Unexpectedly, all of the five generated Mabs were shown to recognize epitopes similar or identical to the RP215-specific epitope. All of the generated RCA Mabs and RP215 were shown to induce apoptosis and CDC of cancer cells in culture [14]. These Mabs were categorized into three distinct groups according to their sequence homology and epitope specificity. All of these Mabs could be paired with RP215 in a typical sandwich enzyme immunoassay [14]. These experimental observations strongly suggest the immunodominance of the RP215-specific carbohydrate-associated epitope. The immunological basis of the immunodominance of RP215-specific "sugar" epitope remains to be explained. It also remains to be demonstrated if differential anti-cancer efficacy exists among the three distinct groups of these biosimilar anti-CA215 Mabs. Due to the existence of the immunodominance of the RP215-associated carbohydrate epitope, we are encouraged to use these RP215 unique epitope(s) or glycolpeptide components for the development of anti-cancer vaccines or antibody-based anti-cancer drugs in humans [14].

\subsection{Anti-Idiotype Monoclonal Antibodies against RP215 as Anti-Cancer Vaccines}

RP215 was shown to inhibit the growth of a variety of cancer cells in vitro and in vivo $[9,11]$. Anti-idiotype (anti-id) Mabs against RP215 were generated in the rat and characterized for future development of epitopespecific anti-cancer vaccines in humans. These rat anti-id Mabs were generated by using $F\left(a b^{\prime}\right)_{2}$ fragments of RP215 as the immunogen. Anti-id Mabs were then utilized for subsequent immunizations in mice to induce anti-anti-id (Ab3) antibody responses. By using TUNEL apoptosis assay, both RP215 and Ab3 were shown to induce apoptosis in cultured cancer cells [4, 7-9,11,13]. Ab3 and RP215 were shown to react similarly with cancer cells from cell lines of different human tissue origins by immunohistochemical staining assay and by Western blot assay [15].

Results of this study suggest that anti-id Mabs bear the properties of the internal image of RP215-specific carbohydrate-associated epitope [11]. Ab3 immune responses can be induced upon immunization of rat anti-id Mab in mice. Ab3 were shown to have immunological properties similar to that of RP215 in terms of the efficacy to induce apoptosis to cancer cells. Therefore, anti-id Mabs against RP215 could be a good candidate for the development of anti-cancer vaccines in the future [11].

\section{DISCUSSION}

When RP215 Mab was initially generated against the OC-3-VGH ovarian cancer cell extract in 1987, it was initially used as for diagnostic applications to monitor serum levels of CA215, a pan cancer biomarker among cancer patients $[16,17]$. It was later shown that the epitope recognized by RP215 was carbohydrate-associated and reacts with CA215, which consists mainly of IgSF proteins. Cancerous immunoglobulins are widespread among all cancer cells and have been demonstrated to be essential for the growth/proliferation of cancer cells $[4,8,11]$. Therefore, RP215 may become a unique probe to target surface bound immunoglobulins and serve as antibody-based anti-cancer drugs for therapeutic applications [11]. In this review, efforts were made to summarize the characteristics of this carbohydrate-associated "sugar" epitope recognized by RP215 Mab, through peptide mappings and glycoanalysis, demonstration of immunodominance, and generation of anti-id Mabs against RP215 [1,11]. The progress of this research is briefly described in this review for the potential development of "sugar" epitope-based anti-cancer vaccines. With RP215 and anti-human IgG as the affinity ligands in two-step immunoaffinity chromatography, cancerous $\operatorname{IgG}$ was 
purified from the shed media of two separate cancer cell lines, OC-3-VGH (ovary) and C33A (cervix). Two Olinked oligosaccharides were identified from the purified cancerous IgG, but were absent in normal human IgG $[1,12]$. It was assumed that these O-linked oligosaccharides may be related to the RP215-specific carbohydrate-associated epitope in cancerous IgG. However, this assumption can be verified only when the primary structure of the carbohydrate moiety is completely elucidated [1].

The immunodominance of the RP215-specific carbohydrate-associated epitope is an interesting phenomenon $[14,18]$. When mixtures of purified CA215 glycoproteins, each of which carries the RP215-specific epitope, were used as immunogen to generate Mabs, all the recovered clones were found to secrete Mabs recognizing epitope(s) similar to that of RP215 [14]. This observation indicates the immunodominance of the RP215-specific "sugar" epitope, which may have potential application in future cancer vaccine development $[14,18]$.

Attempts were made to demonstrate the generations of rat generated anti-id Mabs against RP215 for cancer vaccine development [11]. Following generations of monoclonal anti-id antibodies in the rat, anti-id antibodies can be mass-produced and serve as the immunogen in animals or humans to induce Ab3 responses. Experimental evidence has indicated that $\mathrm{Ab} 3$ antiserum is similar to RP215 in recognizing the RP215-specific epitope of CA215, with the anti-cancer efficacy demonstrated in our studies, such as through induced apoptosis assays $[4,11]$. Therefore, monoclonal anti-id antibodies can serve as the immunogen for anti-cancer vaccinations to induce Ab3 responses in humans for the therapeutic applications of human cancer [11]. Cancer treatment with anti-id antibodies through active immunizations in vaccinations has been reported previously for leukemia, but with limited efficacy [19]. Vaccination can elicit strong and specific humoral responses to relevant tumor associated antigens, but clinical implications of these results remain fully elucidated. Judging from numerous on-going preclinical studies, the RP215-specific carbohydrate-associated epitope in cancerous immunoglobulins appears to be a suitable candidate for cancer vaccine development $[8,11]$. Furthermore, the carbohydrate-associated epitope-based anti-cancer vaccines can potentially target cancer cells of many tissue origins in humans [11].

\section{CONCLUSION}

There is no doubt that cancerous immunoglobulins play an important role in the growth/proliferation of cancer cells. The carbohydrate-associated epitope(s) on CA215 exhibit their universal expression in different cancer cell lines and the unique characteristic of immunodominance. The experimental results also suggested the concept of developing a vaccine based on either a humanized RP215 Mab or its internal image (anti-id Mab). More effort is still required to elucidate the structures of the carbohydrate-associated epitope on CA215. These updated experimental observations should advance our current understanding in cancer immunology and provide a novel strategy for the development of cancer vaccines.

\section{ACKNOWLEDGEMENTS}

This work was supported in parts by NRC-IRAP program (\#794354) of Canada. Proof readings and preparations of this manuscript by summer research student, Suefay Liu of McGill University, are acknowledged.

\section{REFERENCES}

[1] Lee, G. and Azadi, P. (2012) Peptide mapping and glycoanalysis of cancer cell - expressed glycoproteins CA215 recognized by RP215 monoclonal antibody. Journal of Carbohydrate Chemistry, 31, 10-30.

doi:10.1080/07328303.2011.626544

[2] Lee, G., Laflamme E., Chien, C.-H. and Ting, H.H. (2008) Molecular identity of a pan cancer marker, CA215. Cancer Biology and Therapy, 7, 2007-2014.

http://www.landesbioscience.com/journals/cbt/article/698 4/ doi:10.4161/cbt.7.12.6984

[3] Lee, G., Cheung, A.P., Li, B., Ge, B. and Chow, P.-M. (2012) Molecular and immuno-characteristics of immunoglobulin-like glycoproteins in cancer cell-expressed biomarker, CA215. Immunological Investigations, 41, 429-446.

http://informahealthcare.com/doi/abs/10.3109/08820139. 2012.661007 doi: $10.3109 / 08820139.2012 .661007$

[4] Lee, G. and Ge, B. (2010) Inhibition of in vitro tumor cell growth by RP215 monoclonal antibody and antibodies raised against its anti-idiotype antibodies. Cancer Immunology, Immunotherapy, 59, 1347-1356. doi:10.1007/s00262-010-0864-7

[5] Kimoto, Y. (1998) Expression of heavy-chain constant region of immunoglobulin and T-cell receptor gene transcripts in human non-hematopoietic tumor cell lines. Genes, Chromosomes and Cancer, 22, 83-86. doi:10.1002/(SICI)1098-2264(1998)22:1<83::AID-GCC1 2>3.0.CO;2-O

[6] Qiu, X., Zhu, X., Zhang, L., Mao, Y., Zhang, J., Hao, P., et al. (2003) Human epithelial cancers secrete immunoglobulin $G$ with unidentified specificity to promote growth and survival of tumor cells. Cancer Research, 63, 6488-6495.

http://cancerres.aacrjournals.org/content/63/19/6488.abstr $\underline{\mathrm{act}}$

[7] Tang, Y., Zhang, H. and Lee, G. (2013) Similar gene regulation patterns for growth inhibition of cancer cells by RP215 or anti-antigen receptors. Journal of Cancer Science and Therapy, 5, 200-208. doi:10.4172/1948-5956.1000207

[8] Lee, G., Cheung, A., Ge, B., Zhu, M., Giolma, B., Li, B., et al. (2012) CA215 and GnRH receptor as targets for 
cancer therapy. Cancer Immunology, Immunotherapy, 61, 1805-1817.

[9] Lee, G., Zhu, M., Ge, B. and Potzold, S. (2012) Widespread expressions of immunoglobulin superfamily proteins in cancer cells. Cancer Immunology, Immunotherapy, 61, 89-99. doi:10.1007/s00262-011-1088-1

[10] Lee, G. and Liu, S. (2013) Roles of cancerous antigen receptors and CA215 in the innate immunity of cancer cells. Current Immunology Reviews, In press.

[11] Lee, G., Cheung A.P., Ge B., Zhu M., Li, P.P., Hsu, E., et al. (2010) Monoclonal anti-idiotype antibodies against carbohydrate-associate epitope for anti-cancer vaccine development. Journal of Vaccines and Vaccination, 1, 1-7.

[12] Arnold, J.N., Wormald, M.R., Sim, R.B., Rudd, P.M. and Dwek, R.A. (2007) The impact of glycosylation on the biological function and structure of human immunoglobulins. Annual Review of Immunology, 25, 21-50. doi:10.1146/annurev.immunol.25.022106.141702

[13] Lee, G. and Ge, B. (2009) Cancer cell expressions of immunoglobulin heavy chains with unique carbohydrate-associated biomarker. Cancer Biomarkers, 5, 177188.

[14] Lee, G., Zhu, M., Ge, B., Cheung, A.P., Chien, C.-H., Chow, S.-N., et al. (2012) Carbohydrate-associated immunodominant epitope(s) of CA215. Immunological Investigations, 41, 317-336. http://informahealthcare.com/doi/abs/10.3109/08820139. 2011.633141 doi: $10.3109 / 08820139.2011 .633141$

[15] Lee, G., Ge, B., Huang, T.-K., Zheng, G., Duan, J. and Wang, I.H.Y. (2009) Positive identification of CA215 pan cancer biomarker from serum specimens of cancer patients. Cancer Biomarkers, 6, 111-117. http://dx.doi.org/10.3233/CBM-2009-0134

[16] Lee, C.Y., Chen, K.W., Sheu, F.S., Tsang, A., Chao, K.C. and $\mathrm{Ng}$, H.T. (1992) Studies of a tumor-associated antigen, COX-1, recognized by a monoclonal antibody. Cancer Immunology, Immunotherapy, 35, 19-26. doi:10.1007/BF01741050

[17] Lee, G. (2009) Cancer cell-expressed immunoglobulins: CA215 as a pan cancer marker and its diagnostic applications. Cancer Biomarkers, 5, 137-142.

[18] Wilson, E.H. and Hunter, C.A. (2008) Immunodominance and recognition of intracellular pathogens. Journal of Infectious Diseases, 198, 1579-1581. http://jid.oxfordjournals.org/content/198/11/1579.short doi: $10.1086 / 593020$

[19] Zhao, X., Singh, S., Pardoux, C., Zhao, J., His, E.D., Abo, A., et al. (2010) Targeting C-type lectin-like molecule-1 for antibody-mediated immunotherapy in acute myeloid leukemia. Haematologica, 95, 71-78. doi:10.3324/haematol.2009.009811 